\title{
VIRTUOSE »NICHT-MUSIKER «? \\ DER DisKuRS ZU VIRTUOSITÄT, AUTHENTIZITÄT UND SUBVERSION BEIM AUFKOMMEN ELEKTRONISCHER POPMUSIK AM BEISPIEL DES MUSIKEXPRESS - UND SEINE AKTUELLEN NACHWIRKUNGEN
}

\author{
Ambra Cavallaro und Steffen Lepa
}

\section{Einführung: Verlust von Authentizität und Aura durch Elektronik?}

Mit der Verbreitung von elektronischen Instrumenten und Sequenzern in der populären Musik seit den 1970er Jahren sind neue elektronische Genres entstanden, welche die besonderen Klang- und Kompositionsmöglichkeiten solcher »Werkzeuge « stark betonten (bspw. Disco, Synth-Pop, NDW). Von Journalisten wurde der elektronischen populären Musik von Anfang an eine maschinelle Ästhetik, mangelnde Virtuosität und das Fehlen politischer Botschaften vorgeworfen. Ihr wurde nachgesagt, einem oberflächlichen massentauglichen und dabei kapitalistischen Utopismus zu folgen sowie dem Verlust der Authentizität bzw. Aura in den Klängen und den damit verbundenen sozialen Praxen zu Vorschub zu leisten (vgl. Garcia 2014: 2f.). Ziel des Beitrags ist es zu untersuchen, wie es zu solchen weitverbreiteten Zuschreibungen insbesondere im westdeutschen musikjournalistischen Diskurs kam und ob diese für zeitgenössische Musiker noch relevant sind. Dabei geht es uns nicht um eigene Definitionen von Virtuosität, sondern darum aufzuzeigen, welche Debatten um die Werte Virtuosität, Authentizität und Subversion geführt worden sind, als diese neuen Musikrichtungen sie provokativ in Frage stellten.

In der ersten Teilstudie wird eine historische Perspektive eingenommen und untersucht, ab wann und in welcher Art und Weise vom westdeutschen Musikjournalismus ein problematisches Verhältnis zwischen elektronisch 
programmierten Klängen und den genannten Werten diskursiv konstruiert wurde.

In der zweiten Teilstudie wird die zeitgenössische elektronische Popmusik (ab etwa 2000) in den Blick genommen, welche heute stark vom Einsatz von Software-Instrumenten und Laptops dominiert ist. Dieser technologische Wandel erlaubt die Verwendung sehr vielfältiger haptischer Interfaces in Performances und damit auch eine Wiederkehr musikalischer Live-Praktiken für die elektronischen Popmusik-Genres, wodurch sich erneute Veränderungen bei der diskursiven Zuschreibung von Authentizität und Virtuosität ergeben könnten (siehe dazu den Beitrag von Lorenz Gilli in diesem Band).

Bevor die beiden empirischen Teilstudien und ihre Ergebnisse dargestellt und interpretiert werden, soll jedoch kurz dargestellt werden, welche musiktechnologischen Veränderungen seit den 1970er Jahren in die Popmusik Einzug hielten und damit eine Auseinandersetzung des Popmusikjournalismus mit Fragen von Virtuosität, Authentizität und Subversion provozierten.

\section{Dimensionen des musiktechnologischen Wandels}

Nach einer anfänglichen experimentellen Phase, in der nur wenige Avantgarde-Komponisten (z.B. Karlheinz Stockhausen, John Cage) elektronische Musikinstrumente nutzten, begann in den 1970er Jahren deren Massenvermarktung. Durch die damit ausgelöste zunehmende Verwendung von Synthesizern, Sequenzern und später Samplern wurden viele klangliche und dramaturgische Paradigmen der populären Musik revolutioniert. Diese Studie konzentriert sich vorwiegend auf drei Dimensionen solcher kulturbezogenen Veränderungen: Komposition, Performance und Identität.

Synthesizer und Sequenzer erweiterten erstens die musikalischen Ausdrucksmöglichkeiten immens, wodurch gänzlich neue Kompositionstechniken und Klangdimensionen jenseits von Jazz, Rock und Schlager entstanden. Die ersten anwenderfreundlichen Synthesizer-Modelle (etwa Moog) verliehen den Musikern die Möglichkeit, hunderte verschiedenste Instrumentenklänge mit einer einzigen Tastatur abzurufen und in Echtzeit zu steuern und zu beeinflussen oder auch ganz neue synthetische Klänge zu erzeugen (Pinch/ Bijsterveld 2003: 547). Außerdem wurde mit den Sequenzern eine noch größere Innovation eingeführt: die Möglichkeit einzelne Klangsequenzen aufzunehmen, nachträglich zu manipulieren und in beliebiger Reihenfolge in einem beliebigen Tempo in Musikstücke einzubauen. Dadurch war es Musikern möglich, ihre komplexen kompositorischen Ideen sofort und unabhängig von 
ihren eigenen instrumentalen Fähigkeiten erklingen zu lassen, letztlich sogar die Grenzen jedweden menschlichen Könnens zu überschreiten und Melodien und Rhythmen zu generieren, die von Instrumentalisten bis zu diesem Zeitpunkt unmöglich zu erzeugen waren. Somit verschmolz einerseits die Rolle des Komponisten mit der des aufführenden Musikers und der des Toningenieurs zum »Musikproduzenten «, andererseits entstanden auch völlig neue künstlerisch-musikalische Formen und Klangwelten. Zusammengenommen musste die Verbreitung von Synthesizern und Sequenzern notwendigerweise Einfluss auf die Bewertung von Virtuosität und Schönheit von Musikkompositionen haben (Collins/Schedel/Wilson 2013: 21-23).

Insbesondere Sequenzer haben zweitens aber gleichzeitig auch das Verständnis von Musikperformance geändert, da die prinzipielle Programmierbarkeit der gesamten musikalischen Aufführung den Musikern nun den Freiraum verlieh, nicht alles hörbare Material während der Aufführung durch eigene körperliche Handlungen hervorrufen zu müssen. Die Bühnenbewegungen der Musiker wurden somit - zum ersten Mal in der Musikgeschichte - in Teilen unabhängig vom Instrumentalspiel. Das unmittelbare historische Ergebnis war allerdings eine auffallende Unbeweglichkeit der performenden Musiker, die zunächst noch keine Strategien des Umgangs damit entwickelt hatten (»Impossible Performance «) - was oft als »unvirtuos« bezeichnet wurde (ebd.: 21-23). Dieser Aspekt verursachte kontroverse Debatten bezüglich der Authentizität von Live-Veranstaltungen, da bisher z.B. in der Rockmusik der Virtuositäts-Maßstab für Konzerte gewissermaßen proportional zum Niveau der (Gitarren-)Soli war. Die komplette Abwesenheit solcher Elemente in entsprechenden Aufführungen elektronischer Popmusik destabilisierte den bislang existierenden Begriff von »Live - sowie die Position seiner ideologischen Vertreter (Auslander 1999: 10). Gleichzeitig entstand ein neues Verständnis von Virtuosität, im Sinne der gelungenen Erzeugung auratischer Momente, die sich in einem ekstatisch tanzenden Publikum versinnbildlichte, welches zusätzlich zu den ausführenden Künstlern als Teil der Performance betrachtet und miteinbezogen wurde (Collins/Schedel/ Wilson 2013: 21-23). Somit förderten die neuen Instrumente auch das Entstehen neuer »Kultorte« (Discos und Clubs) für Leute mit ähnlichen Idealen und ähnlichem Musikgeschmack (Thornton 1996: 3).

Eine dritte Umgestaltung vormaliger populärmusikalischer Paradigmen die mit der Verbreitung von Synthesizern und Sequenzern einherging betrifft die Identität der Musiker und das Verhältnis von »Mainstream « und »Subversion«. Vormals war es nur bereits etablierten Künstlern möglich gewesen, sehr aufwändige, klanglich und kompositorisch komplexe Werke zu schaffen, aufzuführen und zu verbreiten. Punk hatte in dieser Hinsicht eine Gegenbe- 
wegung dargestellt, welche sich bewusst subversiv von diesen »Kulturindustrien « absetzte und auf »Handgemachtes« als Ausdruck einer politischen Attitüde gegen den Mainstream setzte. Diese einfache Opposition und damit verbundene öffentliche Zuschreibungen wurden nun hinfällig, als kostengünstige elektronische Musikinstrumente aus Fernost nach einiger Zeit für jedermann erschwinglich waren und komplexe und klanglich aufregende Musikkompositionen im Heimstudio ermöglichten. Gleichzeitig entstand durch jene Preispolitik ein Zugang zum Musikschaffen auch für Angehörige benachteiligter Schichten und Milieus, die sich vormals keine kostenintensive Musikausbildung oder den Erwerb kostenträchtiger Musikinstrumente hätten leisten können. Populäre Musik war nun prinzipiell von jeder Person produzierbar, die entsprechende Geräte besaß, und nicht wie vormals ausschließlich von einer kleinen, musikgebildeten und gut vernetzten und geförderten Elite. Somit wurden durch die Verbreitung elektronischer Instrumente auch bisherige journalistische Zuschreibungen von »Gegenkultur « und »Massenkultur« hinfällig.

\section{Teilstudie 1: Diskursanalyse der Musikexpress-Ausgaben (1975-1984)}

Welche Folgen hatten die im vorangegangen Kapitel dargestellten Herausforderungen musikalischer Kompositions-, Aufführungs- und Identitätspraktiken nun für den popmusikjournalistischen Diskurs? Dieser Frage widmet sich die im Folgenden dargestellte empirische Teilstudie, die analysiert, wie der Mainstream-Popjournalismus in den 1970er und 1980er Jahren in Deutschland auf die Verwendung von Synthesizern und Sequenzern reagierte.

Laut Michel Foucault (1972) stellen Diskurse, genau wie das Medium Sprache selbst, soziale Praktiken dar. Diese zirkulieren nicht einfach frei in der Gesellschaft, sondern verschiedene sozialen Institutionen trachten danach, ihre Macht und ihr Subversionspotential durch bestimmte Mechanismen und Prozeduren ${ }^{1}$ einzuschränken (ebd.: 4f.). Foucaults Werk bietet einen theoretischen Zugang, der zu verstehen hilft, wie in und mit Diskursen soziokulturelle Konventionen und Wertmaßstäbe geformt werden. Die nachfolgende Analyse zielt auf die systematisch-interpretative Enthüllung des

1 Unterschieden werden hier interne (Klassifizierung, Regulierung in Form von Kommentaren, Disziplinierungen, oder ökonomisch-kultureller Verknappung der sprechenden Subjekte) von externen Prozeduren (Verbote - in Form von Tabu oder der Etablierung von Kontrasten wie Vernunft/Wahnsinn, wahr/falsch). 
Zusammenhangs zwischen sozialen Strukturen und institutionellen Konventionen. Diese bezieht neben der Herausarbeitung und Interpretation von expliziten und impliziten sprachlichen Legitimations- und Delegitimationsstrategien im massenmedialen Diskurs oft auch extra-linguistische Elemente (bspw. soziale Kontexte und intertextuelle Verweise) in die Analyse ein.

Das analysierte Datenmaterial besteht aus 50 Beiträgen (Interviews und Artikeln) der Zeitschrift Musikexpress über Popmusiker und ihre erschienenen Platten und Aufführungen, die zwischen den Jahren 1975 und 1984 ihr Debüt mit elektronisch programmierbaren Instrumenten hatten. Diese Gruppe von Musikern wird der Einfachheit halber im Folgenden als EPM-Musiker bezeichnet (EPM für »elektronische programmierte Popularmusik«), unabhängig davon, welche musikalische Genrezugehörigkeit ihnen konkret im populärkulturellen Diskurs zugeschrieben wurde. In die eigentliche Diskursanalyse kamen dabei nur solche Auszüge aus den Beiträgen, in denen entweder von den Journalisten oder von den Musikern die Thematik EPM - der Gebrauch von Synthesizern, Sequenzern oder ähnlichen elektronisch programmierbaren Instrumenten - direkt und eindeutig wertend aufgegriffen wurde (d.h. keine Rezensionen oder Retrospektiven). Dies waren in der Regel interviewartige Passagen. Der Zweck dieses methodischen Vorgehens war es, eine direkte Gegenüberstellung der Haltungen und Provokationen der Journalisten bezüglich der EPM-Thematik und den jeweiligen Reaktionen der Musiker vorzunehmen.

Der monatlich erscheinende Musikexpress wurde für die Analyse als Zeitschrift ausgewählt, da er trotz seiner Ursprünge als holländisches Szenemagazin in seiner in Deutschland vertriebenen und kommerziell relativ erfolgreichen Version niemals als ein Untergrundmedium agierte, sondern von Anfang an am Massengeschmack und einem traditionellen Journalismus- und Musikverständnis orientiert war (Hildebrandt 1980: 227). Die Zeitschrift bekam 1984 mit dem Schweizer Verleger Marquardt eine neue Führung und eine andere programmatische Ausrichtung, da sie mit der Zeitschrift Sounds zusammengelegt wurde (Dax 2013: 3). Für die interessierende Epoche wurde sie als Materialquelle eingesetzt, um insbesondere die Annäherung des Mainstream-Musikjournalismus an den technologisch bedingten musikkulturellen Wandel nachvollziehen zu können.

Die Dekade 1975 bis 1984 wurde ausgewählt, da ab Mitte der 1970er Jahre elektronische Instrumente (bspw. asiatische Synthesizer) zunehmend in der Popmusik verwendet wurden. Das Analyseende wurde auf 1984 festgelegt, da zu diesem Zeitpunkt die Popularität von EPM-Musikstilen in Gestalt der »Neuen Deutschen Welle« ihren Höhepunkt fand und sich der Fokus 
der Berichterstattung, auch durch die Zusammenlegung mit Sounds, nun deutlich veränderte.

Der Ablauf der Analyse erfolgte nach der CDA-Methodik (CDA für Critical Discourse Analysis) von Norman Fairclough (1989) und Jean Paul Gee (1999), die drei Schritte beinhaltet:

1 Descriptive Stage: Bei diesem ersten Schritt werden die formalen Eigenschaften des sprachlichen Diskurses (Wörter, Text, Struktur, Grammatik) analysiert. Hierbei ging es darum, mit welchen Begriffen, Argumenten und Phrasen Virtuosität, Authentizität und Subversion von Journalisten und Musikern verhandelt und (de-)legitimiert wurden.

2 Interpretative Stage: In der zweiten Phase wird untersucht, welche Interaktionsstrukturen im Diskurs vorliegen und wie Deutungshoheit hergestellt wird. Zu diesem Zweck wurden im Material besonders die performativen Interaktionen zwischen Künstlern und Journalisten analysiert, die sich in den abgedruckten Interviewauszügen dokumentierten, und die dabei eingesetzten Sprechakte, rhetorischen Schemata und Sprechrollen untersucht.

3 Explicative Stage: Im letzten Schritt der Analyse wird versucht zu verstehen, wie sich vermittels des Diskurses Machtverhältnisse auf der institutionellen Ebene aufbauen und welche historische Ursachen hinter den verwendeten Legitimierungsstrategien stecken. Dazu wurde in der vorliegenden Studie der Zusammenhang zwischen den analysierten linguistischen Interaktionen und den dahinterstehenden institutionellen sozialen Kontexten (Pop-Journalismus, Tonträgerindustrie) betrachtet, um deren engen Bezug begreifen zu können.

\subsection{Teilstudie 1: Materialüberblick}

Im Folgenden wird ein Überblick des der Diskursanalyse zugrundeliegenden Materials gegeben. Tabelle 1 zeigt die Anzahl der Beiträge, die vom Musikexpress während der betrachteten Dekade das Thema EPM zum Gegenstand hatten. Im Jahr 1975 wurde noch kein Beitrag zu EPM veröffentlicht, obwohl elektronisch programmierbare Instrumente bereits langsam dank Musikern wie Kraftwerk populär wurden. Die ersten Artikel erscheinen dann ab der zweiten Hälfte des Jahres 1976 in

\begin{tabular}{|c|c|}
\hline Jahr & Beiträge \\
\hline 1975 & 0 \\
1976 & 2 \\
1977 & 3 \\
1978 & 5 \\
1979 & 3 \\
1980 & 5 \\
1981 & 6 \\
1982 & 20 \\
1983 & 3 \\
1984 & 3 \\
\hline
\end{tabular}

Tabelle 1: Datenbasis 
Form eines vierteiligen Dossiers mit dem Titel »Musik aus der Steckdose . In jeder Folge werden hier ein bestimmter innovativer Aspekt der elektronisch-populären Musikstile detailliert erläutert, die jeweiligen Pioniere vorgestellt und ein Glossar mit den wichtigsten Begriffen für den Aufbau eines »richtigen« Wortschatzes zum Thema geboten.

In der Zeitspanne von 1977 bis 1981 bleibt die Anzahl der veröffentlichten Artikel ziemlich konstant und beschränkt, mit einer leichten Tendenz zur Steigerung während der letzten Jahre. 1982, als die so genannte »Neue Deutsche Welle (NDW) die Spitze ihres kommerziellen Erfolgs erreichte, findet sich plötzlich eine auffallend große Anzahl an Beiträgen, die das Auswahlkriterium erfüllten. Außerdem erscheinen ab 1982 zwei neue Rubriken in Musikexpress mit den Titeln »Deutsche Tänze « und »Neue Gesichter «, die jedoch 1983 wieder abgesetzt werden. Parallel nimmt auch die explizite Auseinandersetzung mit elektronisch-populärer Musik wieder deutlich ab.

\begin{tabular}{|l|l|l|}
\hline \multicolumn{2}{|c|}{ Betrachtete Musiker (Jahr des Debüts mit EPM-Instrumenten) } \\
\hline ABC (1983) & Gary Numan (1979) & O.M.D. (1980) \\
Alan Parsons (1977) & Giorgio Moroder (1979) & Palais Schaumburg (1981) \\
Alan Vega (1980) & Heaven 17 (1981) & Pere Ubu (1978) \\
Ash Ra Tempel (1976) & Holger Czukay (1979) & Pete Schelley (1981) \\
Au Pairs (1982) & Human League (1979) & Peter Gabriel (1977) \\
B.E.F. (1980) & Japan (1978) & Phil Collins (1980) \\
Bananarama (1983) & Jean Michel Jarre (1973) & Siouxsie \& The Banshees \\
Brian Eno (1975) & John Foxx (1980) & (1979) \\
Classic Nouveaux (1981) & John Watts (1982) & Soft Cell (1981) \\
Culture Club (1982) & Kim Wilde (1982) & Taco (1980) \\
Cyndi Lauper (1983) & Klaus Nomi (1982) & Talking Heads (1982) \\
D.A.F. (1979) & Klaus Schulze (1972) & Tangerine Dream (1974) \\
Depeche Mode (1981) & Kraftwerk (1970) & Ultravox (1978) \\
Devo (1979) & Level 42 (1981) & Vince Clarke (1980) \\
Die Krupps (1981) & Malaria (1982) & XTC (1979) \\
Eberhard Schoener (1971) & Michael Hoenig (1978) & Yazoo (1982) \\
Fashion (1979) & Nena (1982) & Yello (1980) \\
\hline
\end{tabular}

Tabelle 2: Liste der in den analysierten Beiträgen erwähnten EPM-Musiker

Tabelle 2 zeigt eine Liste der in den analysierten Beiträgen erwähnten Musiker und den Zeitpunkt ihres jeweiligen Debüts mit elektronisch programmierbaren Instrumenten, das für die meisten zu Beginn der 1980er Jahre liegt. In nur wenigen Fällen begann die Aktivität deutlich früher (z.B. Eberhard Schoener, Jean Michelle Jarre, Klaus Schulze und Kraftwerk), diese können somit als >Pioniere< des EPM-Einsatzes gelten. 


\subsection{Teilstudie 1: Ergebnisse}

\begin{tabular}{|c|c|c|}
\hline Jahr & Kern der Kritiken (Journalisten) & Kern der Verteidigungen (Musiker) \\
\hline 1976 & $\begin{array}{l}\text { - mangelnde körperliche Beziehung } \\
\text { zu den Instrumenten } \\
\text { - Synthesizer auf der Bühne (werden } \\
\text { nicht als »echte« Instrumente } \\
\text { identifiziert) }\end{array}$ & $\begin{array}{l}\text { - Bewusstsein der musikalischen } \\
\text { Strukturen vorhanden } \\
\text { - mit elektronischen Instrumenten } \\
\text { muss man sich auf der Bühne auch } \\
\text { konzentrieren } \\
\text { - intellektuelle Musik } \\
\end{array}$ \\
\hline 1977 & $\begin{array}{l}\text { - mangelnde Originalität der EPM- } \\
\text { Musiker } \\
\text { - unüblicher Sound }\end{array}$ & $\begin{array}{l}\text { - stark intellektuelle und struktu- } \\
\text { rierte Musik }\end{array}$ \\
\hline 1978 & $\begin{array}{l}\text { - EPM vorwiegend unterhaltungs- } \\
\text { fokussiert } \\
\text { - bewegungslose Performance der } \\
\text { Musiker } \\
\text { - mangelnde Originalität der EPM- } \\
\text { Musiker }\end{array}$ & $\begin{array}{l}\text { - elektronische Instrumente bieten } \\
\text { endlose Möglichkeiten } \\
\text { - Bewusstsein der musikalischen } \\
\text { Strukturen vorhanden } \\
\text { - elektronische Instrumente haben } \\
\text { nicht zwangsläufig einen »kalten« } \\
\text { Sound } \\
\end{array}$ \\
\hline 1979 & $\begin{array}{l}\text { - EPM stark mit Kulturindustrie ver- } \\
\text { bunden }\end{array}$ & - \\
\hline 1980 & - EPM kalt und gefühllos & $\begin{array}{l}\text { - EPM ist unkonventionell } \\
\text { - öffentliche Meinung in der Musik- } \\
\text { szene oft resistent gegen Verände- } \\
\text { rungen } \\
\text { - EPM bringt Originalität und Innova- } \\
\text { tion }\end{array}$ \\
\hline 1981 & $\begin{array}{l}\text { - EPM-Musiker inkompetent } \\
\text { - EPM-Musiker sind vor allem } \\
\text { Amateure } \\
\text { - EPM-Musiker bringen zu viele } \\
\text { Instrumente auf die Bühne }\end{array}$ & $\begin{array}{l}\text { - EPM führt eine neue Konzeption von } \\
\text { Originalität ein. Musik ist damit } \\
\text { nicht mehr elitär. EPM ist nicht } \\
\text { unbedingt als »kalt« anzusehen } \\
\text { - Entertainment-Charakter ist auch } \\
\text { wichtig } \\
\text { - EPM-Musiker brauchen viele Instru- } \\
\text { mente auf der Bühne, um deren } \\
\text { Klänge zu erstellen } \\
\end{array}$ \\
\hline 1982 & $\begin{array}{l}\text { - die meisten elektronischen Live- } \\
\text { Performances sind vorprogrammiert } \\
\text { - EPM enthält keine politische Mit- } \\
\text { teilung } \\
\text { - mangelnde Virtuosität der EPM } \\
\text { - mangelnde Originalität der EPM } \\
\text { - Programming vs. Composing }\end{array}$ & $\begin{array}{l}\text { - elektronische Instrumente haben } \\
\text { nicht zwangsläufig »kalten« Sound } \\
\text { - EPM-Musiker sind nicht nur Enter- } \\
\text { tainer oder Amateure } \\
\text { - EPM drückt die gesellschaftliche } \\
\text { Gegenwart aus }\end{array}$ \\
\hline 1983 & - & $\begin{array}{l}\text { - EPM kann durchaus politische Mit- } \\
\text { teilungen enthalten }\end{array}$ \\
\hline 1984 & $\begin{array}{l}\text { - Knöpfe drehen vs. Instrumente } \\
\text { spielen } \\
\text { - Automatisierung des kreativen } \\
\text { Prozesses }\end{array}$ & $\begin{array}{l}\text { - elektronische Instrumente bieten } \\
\text { endlose Ausdrucksmöglichkeiten } \\
\text { - Komposition mit elektronischen } \\
\text { Instrumenten erfordert einen } \\
\text { langen kreativen Prozess }\end{array}$ \\
\hline
\end{tabular}

Tabelle 3: Kern der Kritiken/Verteidigungen im untersuchten Material 
Tabelle 3 zeigt schlaglichtartig die zentralen Ergebnisse von Schritt 1 der Diskursanalyse geordnet nach dem zeitlichen Ablauf der zugrundeliegenden Beiträge. Die linke Spalte fasst die jeweils meist verwendeten Provokationsund Kritikargumente der Journalisten zum Thema EPM zusammen, die rechte stellt diesen die häufigsten Reaktionen der Musiker auf solche >Angriffe< gegenüber.

Die Kritiken beziehen sich im anfänglichen Zeitraum meistens auf die elektronische Natur der Instrumente und auf die vermeintlich mangelnde körperliche Beziehung der Musiker zu diesen »Maschinen«:

»ME: Welche physische Beziehung gibt es mit euren Instrumenten? Ist das so wie bei einer Gitarre?

TD: Es ist nur ein optischer Unterschied, du bist unheimlich konzentriert, das ist auch ein physisches Erlebnis, obwohl niemand da unten wahrnimmt das was passiert [...], während du drehst, drückst und hörst. Du wartest ja nicht 5 Minuten lang und dann passiert etwas!« (Schober 1976a).

In diesem Auszug aus einem Interview mit Tangerine Dream wird die Band befragt, wie sich die körperliche Interaktion mit einem Synthesizer »anfühlt«. Die Reaktion lässt sich zunächst als Belehrung interpretieren, die schließlich in einer Art Polemik (»5 Minuten«) mündet, welche typische Vorurteile des Interviewers bezüglich des betrachteten Themas herausstellt und verdeutlicht, dass es sich - genau wie bei Gitarren - durchaus um Instrumente handelt, die »in Echtzeit« und mitunter virtuos bedient werden müssen.

Mit der stärkeren Verbreitung solcher Instrumente verschob sich auch die Art der Kritik der Journalisten auf den abstrakteren Kreativitätsbereich und die Identität der Künstler. Beispiele dafür sind Vorwürfe eines Mangels an Originalität, Authentizität und politischer Haltung sowie der Vorwurf vermeintlicher »Kälte« des Klanges elektronischer Musik. Im folgenden Beispiel wird die Band Die Krupps mit D.A.F. - einer der zu dieser Zeit berühmtesten NDW-Bands - stark mit einem vermeintlich populären Trend verglichen und damit implizit wegen vermeintlicher >Originalitätslosigkeit kritisiert:

»ME: Die neue Band der neuen deutschen Tanzmusik verrenkt sich die Hüfte beim Mussolini ${ }^{2}$. [...] Rund um die monotonen Klangexperimente der Stahlwerk Symphonie 3 hat die Modern-Dance-Truppe ihre Musik für Durchschnittstänzer aufgebaut. [...] Die Krupps haben sich ebenfalls für die Industrie ent-

2 »Der Mussolini«, Titel der Band D.A.F.

3 Debütalbum der Band. 
schieden und präsentieren deren reduziertes Konzept in einem D.A.F-Format, obwohl die Band sich natürlich gegen jeden Vergleich wehrt [...].

DK: D.A.F. sind doch ein Indiz für Ja-Tanzmusik, da diese effektvoll weiterverarbeitet wird.

ME: Aber ihr benutzt doch textliche Imperative, genauso wie D.A.F.!« (Meierding 1982a).

Während der letzten zwei betrachteten Jahre (1983 und 1984) sind Argumente ähnlicher Natur wie in 1975 und 1976 zu finden, die aber diesmal auf eine neue Generation von elektronisch programmierbaren Instrumenten gerichtet sind: Die zu dieser Zeit sich stark verbreitenden MIDI- und SoftwareSequenzer, welche die vormals in Synthesizern eingebauten Step-Sequenzer ablösten und nun das Verschalten und Steuern des gesamten Geräteparks ermöglichten. In der Folge wurden vor allem die damit ermöglichten neuen Kompositionstechniken unter die Lupe genommen und das Programmieren von Musik zusammen mit der neuen Gestaltung der Aufnahme- und Probestudios, ähnlich wie vormals die Verwendung von Synthesizern, mit stark ideologisch aufgeladenen Argumenten abgelehnt.

»ME: Die Muse küsst den Künstler nicht mehr im stillen Kämmerlein, sondern versteckt sich in den Floppy Discs des Fairlight-Computers von Vince Clarke, Mastermind der Gruppen Depeche Mode, Yazoo und Assembly [...]. Hier [im Vince Clarke Studio] wird kein mechanisches Instrument zu Hilfe gezogen, hier wird Musik per Knopfdruck gemacht, hier werden Songs nicht länger geschrieben, sondern programmiert. [...] Das sind das Mischpult, die Aufnahmegeräte, Mikrophone, Lautsprecher, ein Fairlight Computer [...]. Durch eine gläserne Verbindungstür kommt man in dem zweiten Raum, wo normalerweise Musiker ihre Instrumente spielen, welcher in ein Büro verwandelt wurde« (Evert 1984).

In der Einführung des Artikels »Im Studio mit Vince Clarke - Computer Kid« beschreibt der Journalist dessen Tonstudio, das anscheinend komplett aus digitalen Instrumenten besteht. Es wird unterstrichen, dass die Natur dieser Geräte in der Verabschiedung der klassischen Musikkomposition läge, und sprachlich angedeutet, dass dafür keinerlei Vorkenntnisse mehr benötigt würden, da die Musik einfach mit einem Knopfdruck produziert werden könne. Ähnliche latente Kritiken und Provokationen finden sich auch in den Überschriften verschiedener Artikel:

»Wir sind eine Radio Station« (Kraftwerk)

»Laser in Concert« (Eberhard Schoener)

»Die Mensch-Maschine « (Gary Numan)

»Maschinen machen mich zum Menschen« (John Foxx) 
»Magie aus dem Computer« (Holger Czukay)

»Emotion \& Electronic « (Soft Cell)

»Intelligent und tanzbar« (Palais Schaumburg)

In diesen Beiträgen wird der Einsatz von elektronischen Instrumenten seitens der betrachteten Musiker als vorrangiges stilistisches Element jeweils stark hervorgehoben. Manche lassen auch darauf bezogene Kritik anklingen, die später innerhalb des Textes metaphorisch weiter ausgearbeitet wird, wie etwa die häufige Kombination von »Mensch« und »Maschine « oder die semantische Annäherung zwischen Attributen wie »intelligent«/»tanzbar« oder »Gefühle«/»Elektronik«. Die Musiker zeigen ihrerseits unterschiedliche Reaktionsformen. In den häufigsten Fällen versuchen sie den Kritiken argumentativ etwas entgegen zu setzen, wobei sich einige Künstler (meistens die kommerziell erfolgreichen oder Pioniere) auch trauen, die Provokationen mit eigenen Provokationen zu beantworten. Dem nachfolgenden Auszug lässt sich eine entsprechende provozierende Antwort von Sal Solo entnehmen, Sänger der Band Classic Nouveaux, als seine künstlerische Annäherungsweise als $z u$ »entertaining « bezeichnet wird:

»ME: Sal Solo bekennt sich ohne Verlegenheit zum reinen Entertainment für seine erste Konfrontation mit dem Pop-Sound [...].

CN: Der unterhaltende Aspekt ist für mich wichtiger, weil man sich sonst viel zu schnell in eine Sache hineinsteigern kann [...]. Politik und all diese Dinge haben ihren Platz, aber sie sollten nie auf die Musik übergreifen. Wenn du Politiker werden willst, solltest du Politiker und nicht Musiker werden« (Meierding 1981a).

Eine wichtige, mehrfach aufscheinende Strategie zur Verteidigung gegenüber dem Vorwurf mangelnder Virtuosität ist das Reklamieren der betroffenen Musiker (hier: Kraftwerk), trotz des Einsatzes von elektronisch programmierbaren Instrumenten durchaus über ein Bewusstsein für musikalische Strukturen zu verfügen:

»ME: Was passiert, wenn ihr an eurem Synthesizer sitzt?

KW: Du weißt, wenn du Scheiß spielst; Wir wissen auch, wenn wir von AMoll auf D-Dur wechseln. Diese Sachen stehen auch sehr im Vordergrund « (Schober 1976b).

Ähnlich auch in einem Interview mit Michael Hoenig:

»ME: Wie kann jemand, der Musik macht, behaupten, >dass die Grundelemente der Musik für ihn keine Bedeutung haben ?

MH: Dieser Satz ist etliche Jahre alt. Inzwischen hat die Musik das Elektronische etwas zurückgedrängt. Ich habe keine klassische musikalische Ausbil- 
dung. Ich bin natürlich mittlerweile in der Lage, auf dem Klavier Harmonien und Klangstrukturen aufzubauen« (Freund 1978).

Darüber hinaus weisen einige Pioniere wie Brian Eno auch deutlich auf den innovativen Charakter elektronischer Musikinstrumente hin sowie auf die Tatsache, dass die neuen Produktionsformen auch eine »neue« Gesellschaft widerspiegeln würden:

»BE: Bezüglich der neuen Musik, die ich heutzutage höre, möchte ich darauf hinweisen, dass es hier etwas Neues und Anderes gibt, und dass es Zeit wird, eine adäquate Sprache zu entwickeln, um darüber zu sprechen« (In Hülsen 1982b).

\begin{tabular}{|l|l|l|}
\hline Negative Annäherung & Positive Annäherung & Neutrale Annäherung \\
\hline - Bananarama & $\varangle$ Alan Vega & - Classic Nouveaux \\
- B.E.F. & $\varangle$ Brian Eno & $\triangleright$ Eberhard Schoener \\
- Depeche Mode & $\varangle$ Giorgio Moroder & - Heaven 17 \\
- Die Krupps & $\varangle$ Holger Czukay & $\square$ Klaus Schulze \\
- Fashion & $\triangleright$ Jean Michel Jarre & $\square$ Kraftwerk \\
- OMD & $\varangle$ John Foxx & - Palais Schaumburg \\
- Ultravox & $\varangle$ John Watts & - Soft Cell \\
- Yazoo & $\varangle$ Pere Ubu & $\square$ Tangerine Dream \\
& $\varangle$ Pete Schelley & - Yello \\
& \multicolumn{2}{|c|}{ Legende: $\square$ Pince Clarke } \\
\hline
\end{tabular}

Tabelle 4: Typen der diskursiven Annäherung nach Musikertypen

In Tabelle 4 sind die Typen der diskursiven Annäherung der Journalisten nach Musikertypen zusammengefasst. Es ist leicht zu bemerken, wie bei den damals debütierenden Synth Pop-Bands eine negative Annäherung überwiegt (u.a. Depeche Mode, Ultravox!, OMD) und wie anderseits eine positive Annäherung bei klassisch-kompositorisch arbeitenden und etablierten E-Musikern (Jean Michelle Jarre und Brian Eno) wie auch kommerziell erfolgreichen Künstler (u.a. Giorgio Moroder) vorgenommen wurde. Gemischte bzw. neutrale Annäherungsstrategien finden sich vor allem gegenüber den Pionieren der elektronischen Musik (u.a. Klaus Schulze, Kraftwerk und Eberhard Schoener): Auf der einen Seite werden sie tatsächlich schon aufgrund ihres kommerziellen Erfolgs anerkannt, anderseits behalten die Journalisten dennoch einen teils kritischen Unterton bei.

Die nachfolgenden zwei Beispiele zeigen den deutlichen Annäherungsunterschied, den es bei Synth Pop-Musikern im Unterschied zu klassisch ausgebildeten Komponisten gibt. 
»ME: Auf den britischen Inseln sind sie die Stars, Stars für unzählige Fußpaare auf dem Post-Travolta-Tanz-Boden. Stars für die schminkfreudigen New-Romantic-Pfaue, Stars für die Freude der Pop-Musik, aus den Oszillatoren und Filtern des elektronischen Instrumente Parks, der mit jedem Tag größer, besser und billiger wird [...]. [Das Debut-Album] Speak and Spell ist schon in den Charts, eine wunderbare kleine Pop-Praline aus dem Baukasten und noch ein Album im New Wave Fach der örtlichen Schallplattenläden. [...] Habt ihr denn so was wie eine Botschaft? Wollt ihr Leute glücklich machen? DM: Da ist keine Botschaft... Außerdem sind wir keine glücklichen Typen, oder? We're just a fun band!« (Güldner 1982c).

Ganz anders als bei Depeche Mode wird der Gebrauch von Synthesizern beim anerkannten Komponisten Jean Michel Jarre eingeführt:

»ME: Die konventionellen Lehrmethoden hatten ihn jedoch der klassischen Musik entfremdet aber er hat es mit der musikalischen Recherche nicht aufgegeben. [...] Er experimentiert jetzt mit einem Synthesizer.

JMJ: Meine Aufgabe besteht darin, andere zu inspirieren und höhere Vorstellungen und Gefühle zu erzeugen« (Trenkler 1979).

Die dargestellte Einführung des Artikels über Depeche Mode erscheint ziemlich negativ und von latenten Vorwürfen durchzogen, was die Band und ihr erstes Album betrifft. Es wird als ein konstruiertes Supermarkt-Produkt dargestellt, welches gleich jedem anderen Produkt aus dem Regal sein könnte. Völlig gegensätzlich wird demgegenüber der Einsatz von Synthesizern seitens Jean Michel Jarre aufgewertet, etwa mit dem Hinweis darauf, dass er über eine klassische musikalische Ausbildung verfügt. Infolgedessen, so scheint das Beispiel nahezulegen, ist ihm das Experimentieren mit elektronischen Instrumenten erlaubt.

\section{Teilstudie 2: Interviewanalyse (2000er)}

Die Ausdrucks- und Gestaltungsmöglichkeiten elektronisch programmierbarer Instrumente haben sich seit den 1970er Jahren stark weiterentwickelt und damit nicht nur neue Genres hervorgebracht, sondern die Popmusik insgesamt verändert. Bestimmend ist heute die Verwendung von Computern bei der Musikproduktion und Musikperformance, welche nun Software Tools für das Sequencing und die Klangsynthese bereitstellen. Die HardwareInstrumente aus den 1970er und 1980er Jahren stellen für viele nun mehr »kultige« Vintage-Objekte dar, die aber dennoch von einigen Künstlern immer noch in Anspruch genommen werden (Pinch/Reinicke 2009). 
Mit der massiven Einführung des Computers in das Musikschaffen verschwammen ehemalige Unterscheidungen zwischen Hochkultur und Massenkultur sowie zwischen Amateur- und Profimusikern noch stärker, da es nicht einmal mehr spezifischer musikelektronischer Gerätebedarf, um klanglich hochwertigen musikalischen Ausdruck zu erzeugen. Jeder Computerbesitzer kann ein potentieller Remixer oder Produzent werden und hat dazu noch die Gelegenheit, Inspirationen und Austausch innerhalb der Netzwerke und Communities des Internets zu erlangen (Cox 2004: XIV) oder seine Musik losgelöst von klassischen Vertriebsstrukturen der Tonträgerindustrie hier vertreiben.

In der zweiten Teilstudie des vorliegenden Beitrags werden daher Musiker in den Blick genommen, welche diese neue Generation der elektronisch programmierbaren Instrumente verwenden. Ziel ist es herauszuarbeiten, ob und inwiefern der Diskurs bezüglich vermeintlich mangelnder Authentizität, Virtuosität und Subversion von EPM auch für die zeitgenössische Szene eine Bedeutung hat. Dazu wurden drei Leitfaden-Interviews mit gegenwärtig aktiven Künstlern aus unterschiedlichen Bereichen der elektronischen Musik geführt. Diese wurden akustisch-digital aufgezeichnet, anschließend transkribiert und später nach der Grounded Theory von Barney G. Glaser und Anselm L. Strauss (1999) interpretiert. Diese Methode erlaubt es, mit Hilfe der thematisch-hermeneutischen Codierung des Aussagenmaterials ein systematisches Bild von der Weltkonstruktion der betrachteten Akteure zu bekommen.

Die Auswahlkriterien für die zu befragenden Musiker wurden so gestaltet, dass mit einem ziemlich beschränkten Sample eine möglichst umfangreiche Perspektive der heutigen EPM-Landschaft resultieren würde. Aus diesen Überlegungen heraus wurden drei professionelle MusikerInnen befragt, die über mindestens zehn Jahre Erfahrung im Bereich der elektronischen Musikproduktion und -Performance mit dem Computer hatten. Um einen möglichst breiten Blick auf die elektronische Musikszene zu bekommen, wurden drei MusikerInnen ausgewählt, die möglichst kontrastierende ästhetische Strategien vertreten und unterschiedliche Perspektiven zur Frage der Kommerzialität aufweisen sollten: Ein Techno-Produzent (Musiker 1), ein IDM-Produzent ${ }^{4}$ (Musiker 2), und eine experimentelle Klangkünstlerin (Musiker 3). Diese wurden per E-Mail kontaktiert, nachdem nach in Berlin wohnenden MusikerInnen gesucht worden war, welche die dargestellten Kriterien erfüllen könnten.

4 IDM = Intelligent Dance Music, Szene-Bezeichnung für experimentellere elektronische Popmusik-Genres und -Musiker. 
Die Teilnehmerlnnen wurden gleich zu Beginn der auf Englisch durchgeführten Interviews gebeten, sich als qualifiziertes Publikum (und nicht als Musiker) zu betrachten, um ihre Relevanzsetzungen bezüglich der Authentizität und Virtuosität der elektronischen Live-Performance besser herauszuarbeiten und möglichst wenig Selbstrepräsentationsstrategien hervorzurufen. Die resultierenden Aussagen dürfen dennoch nicht als authentischer Selbstausdruck betrachtet werden, sondern müssen weiterhin als eine bestimmte Form der PR-Arbeit gesehen werden, die Aufschluss über die für sie als befragte Musiker bestimmende Diskursordnung gibt und somit den Vergleich mit den Analyseergebnissen der CDA erlaubt.

Die Interpretation der Interviews erfolgte in drei sequenziellen Schritten:

1 Open Coding und Memoing: Bei diesem ersten Schritt werden die wichtigsten Phänomene (im Sinne sprachlicher Konstrukte) innerhalb der gesammelten Daten identifiziert, markiert, in Form von paraphrasierenden Codings verdichtet und ggf. als semantischer Blöcke ähnlichen Bedeutungsgehalts miteinander vernetzt.

2 Selective Coding: Bei dieser Stufe werden aus den identifizierten semantischen Blöcken ein oder mehrere Kernkategorien abgeleitet, welche die Unterkategorien miteinander verbinden. In der konkreten Studie waren dies die aufscheinenden Wertschätzungs- und Legitimations-Dimensionen im Hinblick auf die Forschungsfrage.

3 Integrating, Refining and Writing Up: In dieser Phase wird aus den kodierten Daten eine fundierte Theorie unter Rückbezug auf die Forschungsfrage und Literatur abgeleitet. Im konkreten Fall wurde hier die Integration mit den Ergebnissen der CDA unternommen.

\subsection{Teilstudie 2: Ergebnisse}

In Tabelle 5 sind die Ergebnisse des Selective Codings zusammengefasst. Einige der Standpunkte werden von allen Teilnehmenden geteilt, wie etwa die Auffassung, dass es unabhängig von der Medientechnologie die Möglichkeit gäbe, virtuose und authentische Musik zu produzieren. Trotzdem lassen sich auch Unterschiede innerhalb der betrachteten Dimensionen bezüglich der zugeschriebenen Eigenschaften einer »authentischen « Performance und der darauf bezogenen Prioritäten auf der Bühne bemerken, wie die folgenden Interviewauszüge mit den Interviewten aus Techno und IDM zeigen:

»The medium doesn't really make a difference, [...] important is the output [...] one thing that actually GIVES you energy is when the crowd, let's say, 
when the magic happens, then you find yourself FULL of energy « (Musiker 1 - Techno).

»I mean for me there is no difference between a hardware-based live performance and a computer. For me, what is impressing is the way you can affect my perception, and that's it. Because for me, it is not important what kind of information or setup you have, but the way you can connect all this... [...] but I mean, this can happen via laptop, via hardware... at the end there is no difference, I think « (Musiker 2 - IDM).

Während die Musiker 1 (Techno) und 2 (IDM) sich stärker auf ästhetische und technische Elemente konzentrieren (Feedback des Publikums, Set-Up-Organisation der Instrumente, technisches Knowhow), kann man feststellen, dass die Teilnehmerin 3 (Experimentell) demgegenüber eher auf die Dimension der narrativen Mitteilung fokussiert scheint:

»I don't really care about that [the technological medium], because, for me, what is important is the message that they are sending... if they play live with software or not, that's up to them, I don't get annoyed by it « (Musiker 3 - Experimentell).

Diese Unterschiede spiegeln sich auch in den Interviewaussagen zur Art der Veranstaltungen wider, auf denen die Künstler typischerweise tätig sind: Während als Maßstab für die Unterhaltsamkeit und Attraktivität einer Techno-Veranstaltung ein voller Dancefloor mit tanzendem Publikum genannt wird, fühlt sich laut Angaben der Experimentalkünstlerin das Publikum ihrer Konzerte stärker von künstlerisch kommunizierten, subversiven Mitteilungen angesprochen. Infolgedessen wird den genannten Faktoren die zum Erreichen jeweiliger Ziele notwendig sind, von den Befragten auch ein unterschiedliches Gewicht eingeräumt (für 1 und 2 ist dies eher das technische Knowhow, und für 3 der Aufbau einer musikalischen Dramaturgie die dem Transport der intendierten Botschaft dient).

Nichtsdestotrotz scheinen sich Teilnehmer 1 (Techno) und 2 (IDM) im Laufe des Interviews zu widersprechen, was ihre vorherigen Aussagen betrifft. Obwohl sie, angesprochen auf die eigene Praxis, behaupteten, dass die Auswahl der Instrumente die Virtuosität einer musikalischen Performance und/oder Produktion kaum beeinflussen würde, reagieren sie bei der Thematisierung von Aspekten mangelnder Authentizität bei anderen Musikern überraschend >konservativ $<$ :

»Actually there are many many performances, especially within the dancefloor-oriented domain [that I consider as] boring! I mean, in terms of sound, research, because anyway they reproduce the same drum machine and so on... [When I have to ask to myself if the musician on stage is really playing,] 


\begin{tabular}{|c|c|c|c|}
\hline Kategorien & 1. Techno & 2. Ambient/IDM & 3. Experimental \\
\hline $\begin{array}{l}\text { Definition von } \\
\text { Authentizität }\end{array}$ & $\begin{array}{l}\text { - von Medientechnolo- } \\
\text { gie unabhängig sein } \\
\text { - lange/erfolgreiche } \\
\text { Musikkarriere haben } \\
\text { - Experimentelles + } \\
\text { Innovation kombi- } \\
\text { nieren } \\
\text { - bedeutungsvoller } \\
\text { Selbstausdruck } \\
\text { (kein Mainstream) } \\
\end{array}$ & $\begin{array}{l}\text { - von Medientechnolo- } \\
\text { gie unabhängig sein } \\
\text { - Experimentelles + } \\
\text { Innovation kombi- } \\
\text { nieren } \\
\text { - High Quality- Ästhetik } \\
\text { haben } \\
\text { - Originalität liefern }\end{array}$ & $\begin{array}{l}\text { - von Medientechnolo- } \\
\text { gie unabhängig sein } \\
\text { - lange/erfolgreiche } \\
\text { Musikkarriere haben } \\
\text { - Talent + Kreativität } \\
\text { + Charakter haben }\end{array}$ \\
\hline $\begin{array}{l}\text { Definition von } \\
\text { Virtuosität }\end{array}$ & $\begin{array}{l}\text { - } \text { Multimedialität + } \\
\text { Dekonstruktion } \\
\text { - Publikums-Feedback } \\
\text { und Interaktion } \\
\text { fördern }\end{array}$ & $\begin{array}{l}\text { - Forschung bezüglich } \\
\text { Ästhetik durchführen } \\
\text { - gekonnte Set-Up- } \\
\text { Organisation } \\
\text { - technisches Know- } \\
\text { how } \\
\text { - Improvisation } \\
\end{array}$ & $\begin{array}{l}\text { - Kreativität } \\
\text { - Komposition + } \\
\text { Narration } \\
\text { - musikalisches } \\
\text { Training }\end{array}$ \\
\hline $\begin{array}{l}\text { Definition von } \\
\text { Subversion }\end{array}$ & $\begin{array}{l}\text { - Avantgardismus } \\
\text { - Experimentelles + } \\
\text { Innovation kombi- } \\
\text { nieren } \\
\end{array}$ & $\begin{array}{l}\text { - Avantgardismus } \\
\text { - neue musikalische } \\
\text { Sprache und Instru- } \\
\text { mentennutzung } \\
\end{array}$ & $\begin{array}{l}\text { - politisches Engage- } \\
\text { ment } \\
\text { - Genderfragen adres- } \\
\text { sieren } \\
\end{array}$ \\
\hline $\begin{array}{l}\text { Definition } \\
\text { authentischer } \\
\text { Performance }\end{array}$ & $\begin{array}{l}\text { - Entdeckung + Innova- } \\
\text { tion auf der Bühne } \\
\text { - Qualität des Outputs } \\
\text { - recycelte Objekte } \\
\text { als Instrumente } \\
\text { - DJ-Sets }\end{array}$ & $\begin{array}{l}\text { - Experimentelles + } \\
\text { Innovation } \\
\text { - hybride Natur der } \\
\text { Performance } \\
\text { - inspirierende Perfor- } \\
\text { mance }\end{array}$ & $\begin{array}{l}\text { - Interaktion mit dem } \\
\text { Publikum } \\
\text { - bedeutungsvolle } \\
\text { Inhalte } \\
\text { - Präsenz auf der } \\
\text { Bühne }\end{array}$ \\
\hline $\begin{array}{l}\text { Definition } \\
\text { nicht- } \\
\text { authentischer } \\
\text { Musiker und } \\
\text { Performances }\end{array}$ & $\begin{array}{l}\text { - stetige Wiederholung } \\
\text { erfolgreicher } \\
\text { Patterns } \\
\text { - stark standardisierte } \\
\text { Komposition } \\
\text { - vorprogrammierte } \\
\text { Performance } \\
\text { - zu viele Instrumente } \\
\text { oder Laptop auf } \\
\text { Bühne }\end{array}$ & $\begin{array}{l}\text { - stetige Wiederholung } \\
\text { erfolgreicher } \\
\text { Patterns } \\
\text { - stark standardisierte } \\
\text { Ästhetik } \\
\text { - vorprogrammierte } \\
\text { Performance } \\
\text { - Dancefloor- } \\
\text { Veranstaltungen } \\
\text { - zu starke musiktheo- } \\
\text { retische Annäherung }\end{array}$ & $\begin{array}{l}\text { - standardisierte } \\
\text { Ästhetik } \\
\text { - zu viele Instrumente } \\
\text { auf der Bühne } \\
\text { - mangelnde Bühnen- } \\
\text { präsenz }\end{array}$ \\
\hline $\begin{array}{l}\text { Argumente } \\
\text { pro Software }\end{array}$ & $\begin{array}{l}\text { - Erschwinglichkeit + } \\
\text { Tragbarkeit } \\
\text { - zeitgenössische } \\
\text { Musikwerkzeuge }\end{array}$ & $\begin{array}{l}\text { - Erschwinglichkeit + } \\
\text { Tragbarkeit } \\
\text { - zeitgenössische } \\
\text { Musikwerkzeuge }\end{array}$ & - Erschwinglichkeit \\
\hline
\end{tabular}

Tabelle 5: Selective Coding-Kategorien

intellectually this is actually like an offence, because you are not honest as artist. [...] This often happens when you are booked for Techno events, with artists coming from different background playing on the same stage, [...] I mean, they just push >play« (Musiker 2 - IDM).

Auch der befragte Techno-Artist argumentiert in ähnlicher Weise: 
»I have been disappointed from guys just pushing play on the laptop [...]. I find it more interesting as a performance-charisma to see something else than just a laptop, you know, a laptop is a very important tool, but hm... let's say, there is something that always catches my curiosity when I see something is going without a laptop. [I feel] pretty disappointed, if I understand that something is TOO prepared with very little room for improvisation in the performance, then it becomes uninteresting, then I can just go in a music shop and buy a release of them... [I also feel disappointed] when the approach is too easy. I mean, when I see that it is mostly about like... just pushing play, or... calling it a live performance just because they're playing their own things, that they're producing in the studio... It's like if you see a concert of Daft Punk, probably everything there is prepared « (Musiker 1 - Techno).

Beide Befragten kritisieren Musiker, die ausschließlich mit Laptop und vorab aufgenommenen Sequenzen auftreten. Sie bezeichnen diese als uninteressant und beklagen die mangelnde Virtuosität (»Just push Play«, »boring«), sowohl ästhetisch als technisch. Wo 1 (Techno) diesbezüglich sehr Hardware-orientiert scheint, macht 2 (IDM) hier vor allem einen prinzipiellen Nachteil von Dancefloor-Veranstaltungen aus: beide beziehen sich dabei auch auf die Bewegungslosigkeit von Laptop-Musikern. Musiker dieses Genres seien sich einander sehr ähnlich hinsichtlich der Produktions- und Auftrittsweisen, daher seien sie typischerweise nicht besonders originell.

Im Gegensatz dazu bleibt die 3. Teilnehmerin (Experimentell) konsequent bei ihren vorherigen Aussagen bezüglich der softwarebasierten elektronischen Musikszene. Sie bezieht als einzige nicht die Instrumente in ihre Argumentation bezüglich eines vermeintlichen Mangels an Originalität und Virtuosität der Musiker ein und argumentiert, dass diese nur Werkzeuge darstellten, womit man seine eigene Mitteilung ausdrücken könne. Für sie hängt sich die Legitimität an der tatsächlichen Nutzung seitens der Musiker auf:

»I have a critique about it, because nowadays the people focus a lot on the visual performance, in the sense of having a lot of cables, a lot of weird instruments which look cool, and ok, I agree... you need a lot of work in order to do that, but they just focus on the visual aspect, to look weird, but it doesn't also SOUND weird! There is no narrative in that, it is more the spectacle of using machines which they build up themselves « (Musiker 3 Experimentell).

In jedem Fall sind sich alle drei - aus ihrer Perspektive als Performer einig, dass der Einsatz softwarebasierter Instrumente eine finanzielle Erleichterung gegenüber dem Kauf von Hardware wie etwa einem modularen 
Synthesizer bedeutet. Deswegen erscheinen deren Auswirkungen auf die Virtuosität auch akzeptabel:

»It also depends a lot on the possibilities, because, bringing equipment costs a lot of money and that impacts on your actual earnings, [...] and if you travel just with a laptop, your life is easier« (Musiker 1 - Techno).

»I questioned myself about it because I started playing with the computer, with a laptop, because that was accessible for me. At that time, I didn't have the money for synthesizers « (Musiker 3 - Experimentell).

Die Analyse-Ergebnisse zeigen, dass bestimmte marktorientierte Standpunkte von den interviewten, kommerziell erfolgreichen Musikern aus alternativen und experimentell-explorativen Szenen durchaus geteilt werden und weniger Wert daraufgelegt wird, die dominierenden Regeln zu brechen. Insofern lässt sich behaupten, dass auch in diesen vermeintlich >alternativen Szenen kulturökonomische Wertmaßstäbe zählen, die musikalischen Erfolg mit einer gekonnten Marktpositionierung gleichsetzen.

\section{Integration der Teilstudien: Virtuose Nicht-Musiker?}

Die erste Teilstudie der vorliegenden Analyse demonstrierte am Beispiel der Mainstream-orientierten Musikzeitschrift Musikexpress, wie im Musikjournalismus der Diskurs um Authentizität und Virtuosität elektronisch-populärer Musik ab Mitte der 1970er Jahre begann. Im Falle von Musikexpress zeigten sich die Musikredakteure im betrachteten Zeitraum zunächst widerstrebend, überhaupt über die neuen musikalischen Tendenzen zu berichten und ignorierten diese zunächst. Als diese einen kritischen Grad an kommerziellem Erfolg erreicht hatten, ist die Thematik jedoch auch vom Musikexpress in Form von Dossiers eingeführt worden. Ein wichtiges Element dieser DossierReihe war das angeschlossene Glossar, weil damit eine neue ssprache zwecks Etablierung neuer kultureller Bewertungsmaßstäbe für EPM eingeführt werden konnte.

Nach dieser Vorstellung der neuen Musikstile und der Einführung von darauf abgestimmten Bewertungskriterien wurden von Jahr zu Jahr mehr Künstlerinterviews zum betrachteten Thema veröffentlicht, mit einem Höhepunkt im Jahr 1982. Trotz erhöhter Aufmerksamkeit wurden die meisten debütierenden EPM-Bands im Beobachtungszeitrum von den Journalisten in Interviews und Kommentaren jedoch weiter aufgrund ihrer vermeintlichen Virtuositätslosigkeit explizit und implizit ästhetisch abgelehnt, wohingegen 
kommerziell erfolgreiche oder klassisch-ausgebildete Musiker weitaus mehr Respekt erfuhren. In den letzten Jahren der analysierten Zeitspanne (19831984), als der Einsatz von Synthesizern und Sequenzern zu einem Massenphänomen wurde, verlagerte sich die Kritiken auf die Verwendung einer neuen Generation computergesteuerter MIDI-Instrumente, u.a. wegen der nun möglichen Programmierung der Kompositionen.

Die am häufigsten vorkommenden Vorwürfe der Journalisten gegenüber den Musikern sind der Mangel an Virtuosität und Originalität sowie die (zunächst) bewegungslose Performance. Dazu homologe Elemente und Argumentationsfiguren finden sich interessanterweise auch innerhalb der 30 Jahre später getroffenen Aussagen der interviewten MusikerInnen in der zweiten Teilstudie, dies gilt vor allem für 1 (Techno) und 2 (IDM). Ferner sollte ursprünglich untersucht werden, ob es möglicherweise neue oder andere Diskreditierungsargumente bezüglich der Verwendung elektronischer Instrumente und Programmierung in den kontemporären Szenen gibt, aber es konnten hauptsächlich Übereinstimmungen mit denjenigen der 1970er und 1980er Jahre gefunden werden. Beispiele dafür sind etwa die Auffassung, dass programmierte und Laptop-Performances im Kern nicht virtuos seien (»Just push Play«), und die Abqualifizierung reiner Dancefloor-Veranstaltungen.

Es fielen darüber hinaus gewisse Widersprüche in den Äußerungen der Künstler auf, wenn diese sich bezüglich anderer Musiker äußerten, die in denselben Szenen aktiv sind und die gleichen Musiktechnologienbenutzen wie sie. Aus der Tatsache, dass dieses ausschließlich bei den kommerziell erfolgreichen Musikern auftrat, lässt sich ableiten, dass auch vermeintlich alternative Musikszenen von starkideologisch geprägten diskursiven Rahmen begrenzt und beeinflusst werden.

\section{Gesamtinterpretation: Diskursordnung und Kulturindustrie}

Die vorliegende Studie konnte einen Beitrag zum besseren Verständnis der Interdependenz von Technologieentwicklung, künstlerischen Praktiken und popkulturellen Diskursen leisten, insbesondere im Hinblick auf die Frage nach dem Wandel kultureller Wertschätzungsmaßstäbe und Legitimationsstrategien. Darüber hinaus werfen die Ergebnisse auch Schlaglichter auf die Rolle der Massenmedien in diesem Prozess, wodurch sich deren ideologischer Einfluss auf die Popkultur besser nachvollziehen lässt. 
Aus den Ergebnissen der beiden Teilstudien wird sichtbar, wie der musikjournalistische Diskurs um elektronisch programmierbare Instrumente und die damit verbundenen neuen Musikstile von Anfang an von Marktmechanismen geprägt ist. Offenbar öffnen sich die Rock/Pop-Journalisten des Musikexpress in den 1970er und 1980er Jahren erst auf ökonomischen Druck hin dem Thema und etablieren dann eine neue Diskursordnung, die es erlaubt, etablierte Wertmaßstäbe bezüglich der aufkommenden Fragen der Rolle von Technik und Aura auch im Hinblick auf EPM beantworten zu können. In diesem Prozess adaptieren sie Prinzipien für die Bewertung von Authentizität, Virtuosität und Subversion aus dem Rock, einem damals erfolgreichen Stil populärer Musik, der zudem die Journalisten selbst stark prägte. Es fließen ferner auch kritische linksintellektuelle Diskurspositionen etwa aus dem Werk Adornos (1941 u. 1947) ein, wenn zuweilen etwa die neuen Stile als Produkte der Kulturindustrie und des »Commodity Listening « delegitimiert und Authentizität im Lichte »kalter Elektronik « beschworen wird. Erstaunlicherweise scheint die resultierende Diskursordnung heute, 35 Jahre später, auch innerhalb der kontemporären (und kommerziell erfolgreichen) elektronischen Musik-Szene immer noch teilweise wirksam zu sein, wie die Interviewanalyse zeigen konnte, vermutlich auch wegen der gestiegenen Bedeutung des Musikjournalismus als intermediärer Institution am digitalen Musikmarkt.

Diese Schlussfolgerungen unterliegen freilich gewissen Limitationen: Die erste Teilstudie bezieht sich ausschließlich auf die westdeutsche Musikszene und die Perspektive des Mainstream-Musikjournalismus. Weder wurden andere relevanter Länder betrachtet (bspw. Großbritannien und USA), noch wurden avantgardistische Magazine berücksichtigt. Schließlich wäre es zukünftig auch interessant, den Untersuchungszeitraum der Diskursanalyse bis in die 1990er Jahre hinein zu erweitern und für die Interviewstudie eine größere Stichprobe zu wählen. Die vorliegenden Ergebnisse sollten daher als explorativ verstanden werden und möchten vor allem für weitere empirische Untersuchung historischer Diskursordnungen der populären Musik werben.

\section{Literatur}

Adorno, Theodor W. (1941). »On Popular Music. In: Studies in Philosophy and Social Science 9, H. 1 (New York: Institute of Social Research), S. 17-48.

Adorno, Theodor W. I Horkheimer, Max (1947). Dialektik der Aufklärung. Philosophische Fragmente. Amsterdam: Querido.

Auslander, Philip (1999). Liveness: Performance in a Mediatized Culture. London: Routledge. 
Collins, Nick / Schedel, Margaret / Wilson, Scott (2013). Electronic Music. Cambridge Introductions to Music. Cambridge: Cambridge University Press.

Cox, Christoph (2004). »Introduction: Music and the New Audio Culture.«In: Audio Culture: Readings in Modern Music. Hg. v. Christoph Cox und Daniel Warner. New York: Bloomsbury Academic, S. XIII-XVII.

Dax, Max (2013). »Eines von mehreren möglichen Vorworten.«In: Spex - Das Buch. 33 1/3 Jahre Pop. Hg. v. Max Dax und Anne Waak. Köln: Metrolit, S. 5-12.

Fairclough, Norman (1989). Language and Power. New York: Longman Inc.

Foucault, Michel (1972). L'ordre du Discours. Paris: Gallimand.

Garcia, Luis-Manuel (2014). »Richard Dyer, >In Defense of Disco< (1979).«In: Geschichte der Gefühle - Einblicke in die Forschung, November, https://www. history-of-emotions.mpg.de/de/texte/richard-dyer-in-defence-of-disco-1979 (Stand vom 20.4.2017).

Gee, Jean Paul (1999), An Introduction to Discourse Analysis. Theory and Method. London: Routledge.

Glaser, Barney / Strauss, Anselm (1999). The Discovery of Grounded Theory: Strategies for Qualitative Research. Piscataway, NJ: Adline Transaction (Erstausgabe 1967).

Hildebrandt, Barbara (1980). »Information als Werbung - Werbung als Information. Musikzeitschriften. «In: Porträts der deutschen Presse. Politik und Profit. Hg. v. Michael Wolf Thomas. Berlin: Volker Spiess, S. 227-236.

Pinch, Trevor J./ Bijsterveld, Karin (2003). "Should One Applaud? Breaches and Boundaries in the Reception of New Technology on Music. "In: Technology and Culture 44, Nr. 3 (Juli), S. 536-559.

Pinch, Trevor J. / Reinicke, David (2009). »Technostalgia: How Old Gear Lives on in New Music. In: Sound Souvenirs: Audio Technologies, Memory and Cultural Practices. Hg. v. Karin Bijsterveld und José van Dijck. Amsterdam: University Press, S. 152-166.

Thornton, Sarah (1996). Club Cultures: Music, Media and Subcultural Capital. New England: Wesleyan University Press.

\section{Verzeichnis der analysierten Musikexpress-Artikel}

Es ist leider nicht immer möglich, die vollständigen Namen der verantwortlichen Journalisten zu ermitteln, deswegen wird in der nachfolgenden Liste teils mit $\mathrm{Na}$ menskürzeln gearbeitet (soweit verfügbar), und teils »Anon. « als Autor angeführt. Zum Erleichtern des Auffindens werden ferner die jeweils behandelten Musiker/ Bands in eckigen Klammern genannt. ME = Musikexpress.

Anon. (1976). »Disco Sound: Fließband's Baby, Tanzmusik.«In: ME, Nr. 8 (Aug.), S. $42-45$.

Anon. (1982). (Kein Titel) [Level 42]. In: ME, Nr. 9 (Sep.), S. 16.

Anon. (1976a). »Musik aus der Steckdose 1.« In: ME, Nr. 6 (Juni), S. 16-18.

Anon. (1976b) »Musik aus der Steckdose 2. «In: ME, Nr. 7 (Juli), S. 34-36.

Anon. (1976c). »Musik aus der Steckdose 3.«In: ME, Nr. 8 (Aug.), S. 34-36.

Anon. (1976d). »Musik aus der Steckdose 4.«In: ME, Nr. 9 (Sep.), S. 34-36. 
Bauduin, Wolfgang (1977). »Der Tüfftler aus der Abbey Road « [Alan Parsons]. In: ME, Nr. 11 (Nov.), S. 10.

Bonici, R. (1979). »Die Mensch-Maschine« [Gary Numan]. In: ME, Nr. 9 (Sep.), S. $16-$ 18.

Evert, Manfred (1982). (Kein Titel) [Culture Club]. In: ME, Nr. 11 (Dez.), S. 14.

Evert, Manfred (1984). »Im Studio mit Vince Clarke - Computer Kid« [Vince Clarke]. In: ME, Nr. 5 (Mai), S. 48-50.

Freund, W. (1978). »Blubber, blubber, zisch... Der Elektronik Michael hebt ab!« [Michael Hoenig]. In: ME, Nr. 8 (Aug.), S. 9f.

Gockel, Bernd (1981). »Der Tanz der glücklichen Kühe« [Yello]. In: ME, Nr. 11 (Nov.), S. 28.

Güldner, Ulli (1982a). (Kein Titel) [B.E.F.]. In: ME, Nr. 1 (Jan.), S. 18-20.

Güldner, Ulli (1982b). »Intelligent und tanzbar« [Palais Schaumburg]. In: ME, Nr. 3 (März), S. 24.

Güldner, Ulli (1982c). (Kein Titel) [Depeche Mode]. In: ME, Nr. 5 (Mai), S.16.

Güldner, Ulli (1982d). »Pin up-Pop« [Kim Wilde]. In: ME, Nr. 7 (Juli), S. 36-38.

Güldner, Ulli (1982e). (Kein Titel) [Bananarama]. In: ME, Nr. 8 (Aug.), S. 14.

Gülden, Gitti (1982f). (Kein Titel) [Fashion]. In: ME, Nr. 10 (Okt.), S. 16.

Güldner, Ulli (1982g). (Kein Titel) [Au Pairs]. In: ME, Nr. 11 (Nov.), S. 14.

Güldner, Ulli (1982h). (Kein Titel) [Yazoo]. In: ME, Nr. 12 (Dez.), S. 16.

Hoff, Hansi (1980). »Abschied von Winston« [OMD]. In: ME, Nr. 10 (Okt.), S. 30.

Hospelt, Tom (1982). »John Watts: Going on« [John Watts]. In: ME, Nr. 4 (April), S. 46-47.

In Hülsen, Harald (1978). »Der Sound kommt aus dem Stahlwerk« [Pere Ubu]. In: ME, Nr. 7 (Juli), S. 30f.

In Hülsen, Harald (1979). »Herz aus Glas«. [Siouxsie \& the Banshees]. In: ME, Nr. 4 (April), S. 16-18.

In Hülsen, Harald (1980a). »...Just Drums \& Wires!« [XTC]. In: ME, Nr. 1 (Jan.), S. 20-22.

In Hülsen, Harald (1980b). »Weiße Hypno-Tone« [Human League]. In: ME, Nr. 9 (Sep.), S. 25-27.

In Hülsen, Harald (1982a). (Kein Titel) [Pete Schelley]. In: ME, Nr. 5 (Mai), S. 18.

In Hülsen, Harald (1982b). (Kein Titel) [Brian Eno]. In: ME, Nr. 6 (Juni), S. 36-38.

In Hülsen, Harald (1982c). »Der letzte Rebell« [Alan Vega]. In: ME, Nr. 6 (Juni), S. 46-48.

In Hülsen, Harald (1982d). (Kein Titel) [Malaria]. In: ME, Nr. 11 (Nov.), S. 18.

In Hülsen, Harald (1982e). (Kein Titel) [Japan]. In: ME, Nr. 12 (Dez.), S. 28-30.

Loder, Kurt (1984). (Kein Titel) [Cyndi Lauper]. In: ME, Nr. 7 (Juli), S. 30-33.

Meierding, Gabriele (1977). »Ungeschminkt« [Peter Gabriel]. In: ME, Nr. 3 (März), S. 17.

Meierding, Gabriele (1981a). »Dschingis Kahn der Avantgarde« [Classix Nouveaux]. In: ME, Nr. 7 (Juli), S. 20 f.

Meierding, Gabriele (1981b). »Magie aus dem Computer « [Holger Czukay]. In: ME, Nr. 9 (Sep.), S. 14-16.

Meierding, Gabriele (1981c). »Midlife Crisis einer Band« [Ultravox]. In: ME, Nr. 9 (Sep.), S. 34-36.

Meierding, Gabriele (1982a). (Kein Titel) [Die Krupps]. In: ME, Nr. 3 (März), S. $26 f$.

Meierding, Gabriele (1982b). (Kein Titel) [Klaus Nomi]. In: ME, Nr. 5 (Mai), S. 38f.

Meierding, Gabriele (1982c). (Kein Titel) [ABC]. In: ME, Nr. 9 (Sep.), S. 14.

Mia (1982). »Emotion \& Electronic« [Soft Cell]. In: ME, Nr. 1 (Jan.), S. 14. 
Schober, Ingeborg (1976a). »Ein Traum bekommt Flügel« [Tangerine Dream]. In: ME, Nr. 11 (Nov.), S. 8-10.

Schober, Ingeborg (1976b). »Wir sind eine Radiostation« [Kraftwerk]. In: ME, Nr. 12 (Dez.), S. 12-14.

Schober, Ingeborg (1977). »Neuer Start in London« [Ash Ra]. In: ME; Nr. 7 (Juli), S. 18.

Schober, Ingeborg (1978a). »Laser in Concert« [Eberhard Schoener]. In: ME, Nr. 1 (Jan.), S. 34.

Schober, Ingeborg (1978b). »Die aufrechten Vier« [Talking Heads]. In: ME, Nr. 10 (Okt.), S. 14.

Schober, Ingeborg (1980a). »De-vo Da-da (oder auch >Diewo Dada!<)《 [Devo]. In: ME, Nr. 2 (Feb.), S. 26-28.

Schober, Ingeborg (1980b). »Maschinen machen mich zum Menschen« [John Foxx]. In: ME, Nr. 5 (Mai), S. 34-36.

Schober, Ingeborg (1981a). (Kein Titel) [DAF]. In: ME, Nr. 5 (Mai), S. $28 \mathrm{f}$.

Schober, Ingeborg (1981b). (Kein Titel) [Phil Collins]. In: ME, Nr. 5 (Mai), S. 48.

Schober, Ingeborg (1983). »Mit Swing und Schmalz« [Taco]. In: ME, Nr. 3 (März), S. $22 f$.

Schoeneberger, F. (1984). »Endstation Schlaraffenland « [Giorgio Moroder]. In: ME, Nr. 12 (Dez.), S. 26-29.

Sveboda, Stefan (1983a). (Kein Titel) [Nena]. In: ME, Nr. 5 (Mai), S. 22-26.

Sveboda, Stefan (1983b). (Kein Titel) [Heaven 17]. In: ME, Nr. 8 (Aug.), S. 40.

Trenkler, Winfrid (1978). »Der Magier am großen Moog« [Klaus Schulze]. In: ME, Nr. 11 (Nov.), S. 20-22.

Trenkler, Winfrid (1979). »Der Sound kommt aus dem Knopf « [Jean Michel Jarre]. In: ME, Nr. 3 (März), S. 12-14.

\begin{abstract}
The contribution analyses the rise and development of the West-German journalistic discourse about the use of electronic synthesizers, sequencers, and samplers by pop musicians. A discourse analysis reveals the changes in attributions of virtuosity, authenticity and subversion taking place from the 1970s to the1980s as a result of the new electronic means of artistic expression. A complementing interview analysis reveals that the resulting order of discourse is still relevant for the identity of contemporary electronic music artists.
\end{abstract}

